\title{
Comparison between fish and linseed oils administered orally for the treatment of experimentally induced keratoconjunctivitis sicca in rabbits
}

\author{
Danielle Alves Silva ${ }^{1}$, Gisele Alborgetti $\mathrm{Nai}^{2}$, Rogério Giuffrida ${ }^{3}$, Rafael Cabral Barbero ${ }^{3}$, Jacqueline Marcussi \\ Pereira Kuhn ${ }^{3}$, Andressa Caroline da Silva ${ }^{3}$, Ricardo Henrique Zakir Pereira ${ }^{3}$, Maria Fernanda Abbade ${ }^{3}$, Luiz Felipe \\ da Costa Zulim ${ }^{4}$, Carolina Silva Guimarães Pereira ${ }^{4}$ and Silvia Franco Andrade ${ }^{5, *}$ \\ ${ }^{1}$ Animal Science Post Graduate Program, Oeste Paulista University (UNOESTE), Presidente Prudente, SP, Brazil \\ ${ }^{2}$ Department of Anatomy Pathology, Faculty of Medicine (UNOESTE), Brazil \\ ${ }^{3}$ Faculty of Veterinary Medicine (UNOESTE), Brazil \\ ${ }^{4}$ Resident in Small Animal Medicine of the Veterinary Hospital (UNOESTE), Brazil \\ ${ }^{5}$ Department of Small Animal Medicine of the Veterinary Hospital (UNOESTE), Brazil
}

\begin{abstract}
The objective of this study was to compare the efficacy of two sources of omega 3 and 6, fish oil (FO) and linseed oil (LO), orally administered, alone or in combination, for treating experimentally induced keratoconjunctivitis sicca (KCS) in rabbits. Twenty-eight New Zealand rabbits were used in this study. Seven animals were allocated to the C group (negative control), and KCS was induced in 21 animals by topically applying $1 \%$ atropine sulfate drops for 7 days. Treatment with atropine was maintained throughout the study period (12 weeks). The rabbits were divided into 3 treatment groups containing 7 animals each: FO group, LO group and FLO group (FO and LO). The animals were evaluated using the Schirmer Tear Test I (STT I), Rose Bengal Test (RBT), fluorescein test (FT), tear film break-up time (TBUT), and conjunctival and histopathological analysis. There was a significant increase in STT I and TBUT values in treatment groups, but the increase occurred earlier in the FO group. The results of the RBT and FT were similar among treatment groups, except FT, in the FLO group, negative staining was only in 12 weeks. There was a significant decrease in the number of goblet cells in the FLO group compared with the other groups. The results demonstrated that orally administered of FO and LO improved the clinical signs of KCS. However, improvement occurred earlier in the FO group. Using oils in combination did not provide additional benefits. These results contribute to the future development of new oral formulations as adjuvant therapies for KCS.
\end{abstract}

Keywords: Fish oil, Keratoconjunctivitis sicca, Linseed oil, Omega 3 and 6, Oral.

\section{Introduction}

Keratoconjunctivitis sicca (KCS) is a chronic inflammatory disease that is common in dogs and humans and that result from a deficiency in the production of the aqueous portion of tear film (quantitative deficiency) and/or excessive tear evaporation (qualitative deficiency). It mainly affects the cornea and conjunctiva (Miller, 2008a,b; McGinnigle et al., 2012; Stevenson et al., 2012). In the literature, many therapies for KCS have been described, including tear substitutes, immunosuppressive drugs (e.g., cyclosporine, tacrolimus and pimecrolimus), steroidal and nonsteroidal anti-inflammatory drugs, and topical antibiotics to control secondary infections. Other drugs, such as essential fatty acids (EFAs), mucolytics and pilocarpine, are used as adjunctive therapies (Izci et al., 2002; Grahn and Storey, 2004; Berdoulay et al., 2005; Miller, 2008b; Ofri et al., 2009; McGinnigle et al., 2012; Stevenson et al., 2012). Studies in human medicine and veterinary medicine (Sullivan et al., 2002; Barabino et al., 2003; Rashid et al., 2008; Wojtowicz et al., 2011; Neves et al., 2013) have demonstrated that omega-3 $(\omega-3)$ and omega- 6 $(\omega-6)$ can be used to control dry eye because of the derivation of anti-inflammatory agents from these EFAs. In human medicine, orally administered $\omega-3$ and $\omega-6$ has been shown to be an effective alternative treatment for patients with various forms of tear deficiency and those with Sjögren's syndrome (Sullivan et al., 2002; Rashid et al., 2008; Wojtowicz et al., 2011). The principal anti-inflammatory agents, prostaglandins (PGs), leukotrienes (LTs) and thromboxanes (TXs) of series 1, 3 and 5, are derived from the actions of the enzymes cyclooxygenase (COX) 1 and lipoxygenase (LOX) on EFAs (Barabino et al., 2003). $\omega-3$ is derived from linolenic acid (ALA), eicosapentaenoic acid (EPA) and docosahexaenoic acid (DHA), and $\omega-6$ is derived from linoleic acid (LA), $\gamma-$ linolenic acid (GLA) and dihomo- $\gamma$-linolenic acid (DGLA) (Sullivan et al., 2002; Barabino et al., 2003; Neves et al., 2013). Although $\omega-3$ and $\omega-6$ are found in many foods, such as nuts, cold-water fish, soybean 
seeds, canola oil, olive oil and linseed oil (LO), there are differences in the concentrations of these EFAs between natural sources (Sullivan et al., 2002; Rashid et al., 2008; Wojtowicz et al., 2011). Fish oil (FO) is an important source of $\omega-3$ that also contains essential elements, such as selenium, iodine, and vitamins A, B, $\mathrm{D}$ and $\mathrm{E}$. This oil is obtained from cold-water fish, such as salmon, tuna and herring (Oehlenschläger, 2012). FO is classified as a functional food because it facilitates the prevention and treatment of cardiovascular and neurological diseases, general inflammation, asthma, arthritis, psoriasis, and various types of cancer (Kris-Etherton et al., 2002; Harris, 2004; Mahaffey, 2004; Mozaffarian and Rimm, 2006; Jenkins and Josse, 2008; Rand and Asbell, 2011; Oehlenschläger, 2012). A study that tested the effects of a diet rich in $\omega-3$ (obtained by consuming fish) demonstrated a protective effect against macular degeneration in humans (Chong et al., 2008).

LO, which is obtained from the seeds of the Linum usitatissimum plant, is an important source of $\omega-3$ and $\omega-6$ and has an $\omega-3: \omega-6$ ratio of $3: 1$, which is considered ideal, because higher proportions of $\omega-6$ can lead to the formation of pro-inflammatory mediators, such as those derived from arachidonic acid via the enzyme COX2 (PGE2, PGI2, and TXA2) and mediate pathogenic mechanisms, including the inflammatory response (Barabino et al., 2003). LO exerts an immunomodulatory effect by inhibiting interleukin-1, which itself inhibits the production and release of tumor necrosis factor (TNF) by macrophages (Oomah, 2001; Roncone et al., 2010). It also has an anti-inflammatory effect because it induces the formation of antiinflammatory mediators that are derived from the EFAs $\omega-3$ and $\omega-6$ (Oomah, 2001; Covington, 2004; Roncone et al., 2010). One principal difference between FO and LO is that LO requires ALA to be converted to EPA and DHA, whereas in FO, these essential EFAs are already pre-formed as a result of the ingestion of marine plants, which contain synthesized $\omega-3$, by fish [15]. LO is a source of $\omega-6$, from which DGLA, via COX1, produces PGE1 and TXA1 that are anti-inflammatory mediators (Jenkins and Josse, 2008; Rand and Asbell, 2011). Because FO and LO are sources of EFAs, but with differences in their composition and concentrations, and given that no previous studies have compared the efficacies of these compounds for treating KCS, the aim of this study was to compare the efficacy of orally administering each of these two major sources of $\omega-3$ and $\omega-6$ to treat experimentally induced $\mathrm{KCS}$ in rabbits.

\section{Materials and Methods}

This study was approved by the Ethics Committee on Animal Use (CEUA) of UNOESTE (Protocol No. 955) according to the rules of the Brazilian College of Animal Experimentation (COBEA) and the Federal
Council of Veterinary Medicine of Brazil (CFMV) as well as the Association for Research in Vision and Ophthalmology (ARVO) - Statement for the Use of Animals in Ophthalmic and Vision Research. A total of 28 New Zealand adult female rabbits (Oryctolagus cuniculus) that were aged 18 to 22 months old and weighed between 3 and $4 \mathrm{~kg}$ were obtained from the Central Bioterium of the university. The animals were kept in individual cages, with food and water provided ad libitum. Only animals with normal eyes, as identified using ophthalmic exams (biomicroscopy slitlamp examination, pupillary light reflex test, Schirmer Tear Test I (STT I), tear film break-up time (TBUT) test and fluorescein test (FT) were used in this study. The KCS induction protocol was based on those described in previously published studies (Burgalassi et al., 1999; El-Shazly et al., 2008; Shafaa et al., 2011). It involved applying one drop of $1 \%$ atropine (Atropina $1 \%$ colírio $^{\circledR}$, Legrand, São Paulo, Brazil) three times daily. Atropine is a muscarinic blocker, and because the lacrimal gland is innervated by facial nerve (cranial nerve VII) that has a general somatic efferent fibre within the ear canal, a general visceral efferent fibre acting under parasympathetic control to lacrimal glands (Sgrignoli et al., 2013), topical atropine causes a decrease in tear production and consequent inflammation in a manner similar to that which occurs in KCS (Burgalassi et al., 1999). Atropine was applied to both eyes until a diagnosis of KCS (STT I result $\leq 5$ $\mathrm{mm} / \mathrm{min}$ and/or TBUT $\leq 10$ seconds) was achieved. This took an average of 7 days for all of the rabbits in the KCS induction groups. The protocol was maintained by applying atropine two times daily for 12 weeks.

A positive-control group without treatment was not included because of the regulations of the Committee for Experimentation and Use of Laboratory Animals (CEUA/Brazil) of COBEA and the ethical standards of CFMV/Brazil, which prohibits experiments that cause unnecessary suffering in experimental animals. Because the experiment only evaluated drugs that were administered orally, maintaining a positive-control group by applying atropine over 12 weeks without oral treatment would have caused unnecessary discomfort and pain as a result of the disease, the effects of which are well documented in the literature. In any case, time $\mathrm{T} 1$ was the time prior to treatment, and this time was used as the positive control.

Data were analyzed from both eyes of all 28 rabbits. Seven animals were allocated to the $\mathrm{C}$ group (control), in which KCS was not induced and $1 \mathrm{ml} /$ day of $\mathrm{NaCl}$ $0.9 \%$ solution was orally administered as a placebo. KCS was induced in the remainder of the rabbits, which were then randomly divided into 3 treatment groups containing 7 animals each: an FO group ( $1 \mathrm{~g} /$ day of oral Omega 3 Dog $^{\circledR}$, Organnact, Paraná, Brazil), an LO 
group (1 g/day of oral DryLin ${ }^{\circledR}$, Ophthalmos, São Paulo, Brazil), and an FLO group (FO and LO) (500 $\mathrm{mg} /$ day of oral Dog ${ }^{\circledR}$ Omega $3+500 \mathrm{mg} /$ day of oral of $\left.\operatorname{DryLin}^{\circledR}\right)$. The animals received their respective treatments for 12 weeks. The times at which the instillation of $1 \%$ atropine eye drops occurred were 6:00 AM, 2:00 PM and 10:00 PM, and the times at which the oral administration of oils (FO, LO or both oils) occurred was 8:00 AM. Ophthalmologic examinations were performed using a portable slit lamp (SL15, Kowa, Japan) to identify the presence or absence of conjunctivitis, ocular discharge and corneal opacity, with scores described in Table 1. Ocular examinations and tests were always conducted in the morning (10:00 AM) at the following time points: T0 (before the induction of KCS), T1 (1 week postinduction and prior to treatment), and $\mathrm{T} 2, \mathrm{~T} 4, \mathrm{~T} 8$ and $\mathrm{T} 12$ (1, 2, 4, 8 and 12 weeks post-induction, respectively, with treatment). One masked observer (SFA) evaluated the eye examinations and tests.

Table 1. Clinical scoring system.

\begin{tabular}{|c|c|}
\hline Clinical sign & Score \\
\hline \multirow{4}{*}{ Conjunctivitis } & $0=$ None \\
\hline & $1=$ Mild conjunctival hyperemia \\
\hline & $\begin{array}{l}2=\text { Moderate to severe conjunctival } \\
\text { hyperemia }\end{array}$ \\
\hline & $\begin{array}{l}3=\text { Moderate to severe conjunctival } \\
\text { hyperemia and chemosis }\end{array}$ \\
\hline \multirow{4}{*}{$\begin{array}{l}\text { Ocular } \\
\text { discharge }\end{array}$} & $0=$ None \\
\hline & $1=$ Minor serous discharge \\
\hline & $2=$ Moderate mucoid discharge \\
\hline & $3=$ Marked mucopurulent discharge \\
\hline \multirow{4}{*}{ Corneal opacity } & $0=$ None \\
\hline & $1=<25 \%$ \\
\hline & $2=25-50 \%$ \\
\hline & $3=>50 \%$ \\
\hline
\end{tabular}

The STT I was performed without anesthetic eye drops and used to quantify tear production. Values of $\leq 5$ $\mathrm{mm} / \mathrm{min}$ were considered positive for KCS (Maggs, 2008). The TBUT test, which can be used to determine the stability of the tear film and to check for evaporative dry eye in patients who have a normal quantity of tears but unstable tear film, was also performed. This test can explain dry eye symptoms that result from an imbalance in the composition of tears that may be caused by tears evaporating too quickly or not adhering properly to the surface of the eye. The TBUT test was performed specifically to evaluate the quality of the tear film. Two consecutive TBUT evaluations were performed, and their results were averaged. One drop of 1\% Fluorescein Eye Drops (Fluoresceína 1\% colírio $^{\circledR}$, Allergan, São Paulo, Brazil) was placed into the lower conjunctival fornix. The eyelids were manually blinked one time, and the lids were then held open. A slit lamp with a cobalt blue filter was used to determine the time required for the first dark spot, indicating the break-up of the tear film, to appear on the cornea. Values $\leq 10$ seconds were considered diagnostic for KCS (Wei et al., 2013).

The FT, which reveals irregularities in the corneal epithelium, was performed by instillation of one drop of 1\% Fluorescein Eye Drops (Fluoresceína 1\% colírio $^{\circledR}$, Allergan, São Paulo, Brazil) into the lower conjunctival fornix. After the stain was distributed in the tear film, the cornea was evaluated in quadrants (superior temporal, inferior temporal, superior nasal and inferior nasal). The FT score ranged from 0-4 according to the quadrant, with 0 indicating no stain uptake in any quadrant and 4 indicating uptake in all 4 quadrants (Maggs, 2008). The Rose Bengal Test (RBT) was performed to stain tissue that was devoid of mucus. For these tests, Rosa Bengala 1\% Eye Drops (Rosa Bengala $^{\circledR}$, Ophthalmos, São Paulo, Brazil) were applied prior to anesthetic eye drops. The RBT scores ranged from 0-3: (0) no staining, (1) only the conjunctiva was stained, (2) only the cornea was stained, and (3) both the conjunctiva and the cornea were stained (Maggs, 2008). Conjunctival and corneal cytology were assayed at T0, T1, T4, T8 and T12. After anesthetic eye drops were applied, slides were made from conjunctival cells (upper and lower) and the cornea that were harvested using a sterile swab that was moistened with saline solution. The slides were fixed in alcohol for 5 minutes and then stained using the periodic acid-Schiff (PAS) staining technique (Merck, USA) and hematoxylin (Dolles, São Paulo, Brazil). Hematoxylin-eosin (HE) staining was used to evaluate the morphology of the cytoplasm and the nucleus in the cells, and PAS staining was used to evaluate the presence of mucus in goblet cells. The following parameters were evaluated in the conjunctiva: the numbers of neutrophils, lymphocytes, metaplastic cells and anucleate squamous cells. The following parameters were evaluated in the cornea: the numbers of neutrophils, lymphocytes and anucleate squamous cells. Cell counts were determined in 10 fields (40x) that were approximately $1 \mathrm{~mm}^{2}$ in each slide using an optical microscope (Eclipse E200 ${ }^{\circledR}$, Nikkon, Tokyo, Japan). One masked observer (GAN) evaluated the results of all cytological exams.

At the end of the experiment (T12), the rabbits were euthanized using 2.5\% sodium thiopental (Abbott Laboratories, Chicago, IL, USA) in an intravenous dose of $200 \mathrm{mg} / \mathrm{kg}$ (Neves et al., 2013; Sgrignoli et al., 2013). After transpalpebral enucleation was performed, the eyeball was placed in a $10 \%$ formaldehyde solution (Chemical Kinetics, São Paulo, Brazil) for 24-48 hours. The eyeball was then stored in $70 \%$ alcohol and routinely processed and embedded in paraffin (Dynamic Analytical Reagents, São Paulo, Brazil). Each eyeball was cut in half across the sagittal plane for 
histological processing. Three (5 $\mu \mathrm{m}$-thick) serial sections were obtained from the cornea and conjunctiva, and these were stained separately with $\mathrm{HE}$ (Dolles, São Paulo, Brazil), PAS (Merck, USA) and Masson trichrome (Merck, USA). The parameters that were evaluated in the conjunctiva included the numbers of polymorphonuclear neutrophils (PMNs), mononuclear neutrophils (MNs), and goblet cells; the presence of squamous metaplasia in the epithelium; edema; and vascular congestion (e.g., capillary dilation with an increase in the number of intravascular erythrocytes). In the cornea and the lacrimal gland, the presence of edema or vascular congestion and the numbers of PMNs and MNs were assessed. Cell counts were determined in 10 fields $(40 \mathrm{x})$ that were approximately $1 \mathrm{~mm}^{2}$ on each slide using an optical microscope (Eclipse E200 ${ }^{\circledR}$, Nikkon, Tokyo, Japan). Goblet cell density (cells $/ \mathrm{mm}^{2}$ ) was determined in five high-power fields (40x) on each slide, with each field corresponding to an area of approximately $0.5 \mathrm{~mm}^{2}$. One masked observer (GAN) evaluated the results of all histopathological exams. For the STT I and TBUT variables, the goblet cell density, and the numbers of PMNs and MNs, we used two-way analysis of variance (ANOVA) for paired samples with contrasts by Tukey's method. For the FT and RBT variables, we used the nonparametric Friedman test to compare moments and the Kruskal-Wallis test with contrasts by Dunn's method for comparisons of groups. A significance level of $\mathrm{P}<0.05$ was adopted. The software used for statistical analysis was $\mathrm{R}$ version 3.2.2. (The $\mathrm{R}$ Foundation for Statistical Computing, 2015)

\section{Results}

The clinical signs that were observed are shown in Figure 1. Improvements in signs of conjunctival hyperemia, ocular discharge and corneal opacity were observed in the FO, LO and FLO groups from T1 to T12. Neither vascularization nor corneal pigmentation was observed in any group. There was a significant decrease $(\mathrm{P}<0.05)$ in STT I (Fig. 2A) and TBUT (Fig. $2 \mathrm{~B})$ values in the $\mathrm{LO}$ and FLO groups at T1, T2 and T4, but only at $\mathrm{T} 1$ and $\mathrm{T} 2$ in the FO group. The FT was negative at $\mathrm{T} 0$ in all groups, at $\mathrm{T} 8$ and $\mathrm{T} 12$ in the $\mathrm{FO}$ and LO groups, and at T12 in only the FLO group (Fig. 3A). At all other times, the FT was positive (Fig. 3A). All irregularities of the cornea that arose as a result of KCS were found to be superficial (Fig. 4A). The results of the RBT were similar among all treatment groups (Fig. 3B), and all groups were positively stained (Fig. 4B) in the RBT from T1 to T8 and negatively stained at T0 and T12 (Fig. 3B). We obtained and analyzed 28 cytological samples in each group for a total of 112 samples. In $100 \%$ of the samples, conjunctival and corneal cytology at T0 revealed that there were no anucleate squamous cells or squamous metaplasia in any group (C, FO, LO and FLO). An analysis of conjunctival samples at T1 (Fig. 5A) revealed that 57\% of the samples from the FLO group, 35.7\% from the FO group and $42.8 \%$ from the LO group presented a moderate number of anucleate squamous cells, whereas at $\mathrm{T} 4,78.5 \%, 85.7 \%$ and $92.8 \%$ of the samples in the FO, LO and FLO groups, respectively, presented a moderate number. At T8 and T12, the samples from the FO, LO and FLO groups presented a small number of anucleate squamous cells. Based on the number of anucleate squamous cells that were observed using corneal cytology, the samples from the FO, LO and FLO groups presented a small number of such cells at all time periods. Squamous metaplasia (Fig. 5B) was observed at T1 in the FO, LO, and FLO group samples. In the FLO group, at T1, 7.1\% of the samples exhibited anucleate squamous cells (Fig. 5C), but these were not observed in the other groups. In general, there was no mucus in the evaluated groups except in some animals like at $\mathrm{T} 4$ in a rabbit of FO group (Fig. 5D). We obtained and analyzed 28 histological samples of conjunctiva, 28 of cornea and 28 of lacrimal gland, in each group. Based on an analysis of the histopathology of the conjunctiva, goblet cell density (cells $/ \mathrm{mm}^{2}$ ) (Fig. $6 \mathrm{~A}$ and $6 \mathrm{~B})$, was significantly lower $(\mathrm{P}<0.05)$ in the FLO group (5.4 $\pm 2.2 ; \mathrm{P}=0.0012)$ than in the $\mathrm{C}(8.9 \pm 1.8)$, FO (9.6 \pm 2.1$)$ and LO (10.8 \pm 2.0$)$ groups. A moderate amount of squamous metaplasia was observed in $50 \%$ (14 out of 28 samples) of the FLO samples, and vascular congestion was observed in $28.5 \%$ ( 8 out of 28 samples) of the FLO samples. Edema of the conjunctiva was mild in $21.4 \%$ (6 out of 28 samples) and $35.7 \%$ (10 out of 28 samples) of the FO and LO samples, respectively, and moderate in $50 \%$ (14 out of 28 samples) of the FLO group samples. Based on an analysis of the histopathology of the cornea (Fig. 6C), edema (Fig. 6D) and vascular congestion were mild in $7.1 \%$ ( 2 out of 28 samples), $14.2 \%$ ( 4 out of 28 samples) and $21.4 \%$ (6 out of 28 samples) of the FO, LO and FLO samples, respectively. Based on the histopathology of the lacrimal gland (Fig. 6E), the FO and LO samples displayed no changes, whereas in the FLO group, $35.7 \%$ (10 out of 28 samples) demonstrated vascular congestion, and $7.1 \%$ ( 2 out of 28 samples) exhibited inflammatory infiltrates (Fig. 6F). The neutrophils counts $\left(\mathrm{cells} / \mathrm{mm}^{2}\right.$ ) in the conjunctiva, cornea and lacrimal gland were zero in all of the evaluated groups. The lymphocytes counts were zero in all analyzed structures in the $\mathrm{C}$ group, and in all of the treatment groups, the counts in the cornea were also virtually zero. The lymphocytes counts (cells $\left./ \mathrm{mm}^{2}\right)$ in the lacrimal gland was significantly higher $(\mathrm{P}<0.05)$ in the FLO group $(7.7 \pm 2.2 ; \mathrm{P}=0.0032)$ than in the FO $(2.7 \pm 1.0)$ and LO $(2.9 \pm 1.1)$ groups, and there was no significant difference $(\mathrm{P}>0.05)$ in the lymphocytes count in the conjunctiva between the FO $(1.7 \pm 0.8)$, LO $(2.2 \pm 1)$ and FLO $(2.4 \pm 1.0)$ treatment groups. 

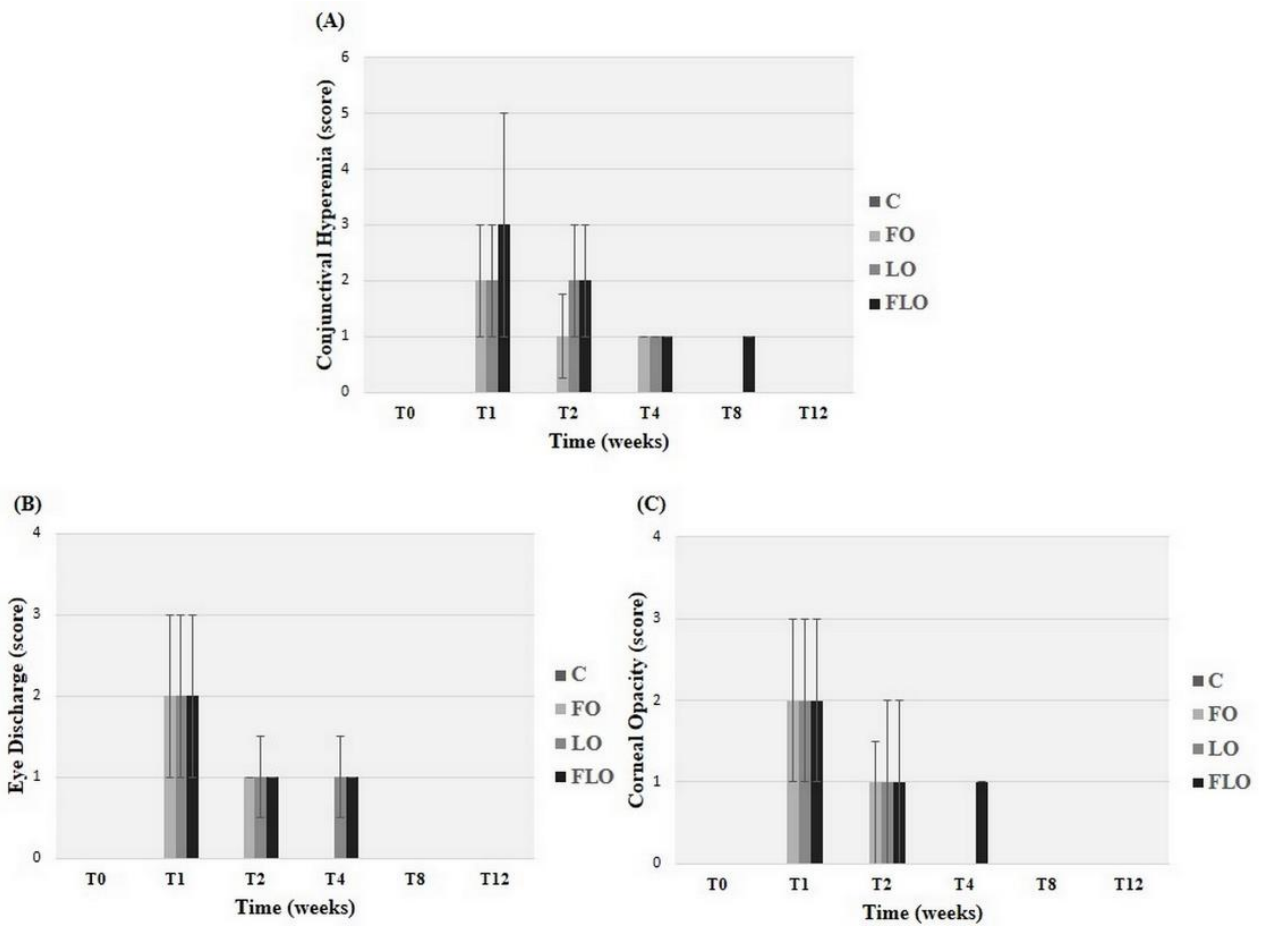

Fig. 1. Medians and interquartile deviations of observed clinical signs (Table 1). (A) Conjunctival hyperemia, (B) eye discharge and $(\mathbf{C})$ corneal opacity in the eyes of rabbits with experimentally induced keratoconjunctivitis sicca (KCS) that were subjected to oral treatment with fish oil (FO), linseed oil (LO), or a combination of fish oil and linseed oil (FLO), and the control group (C).

(A)

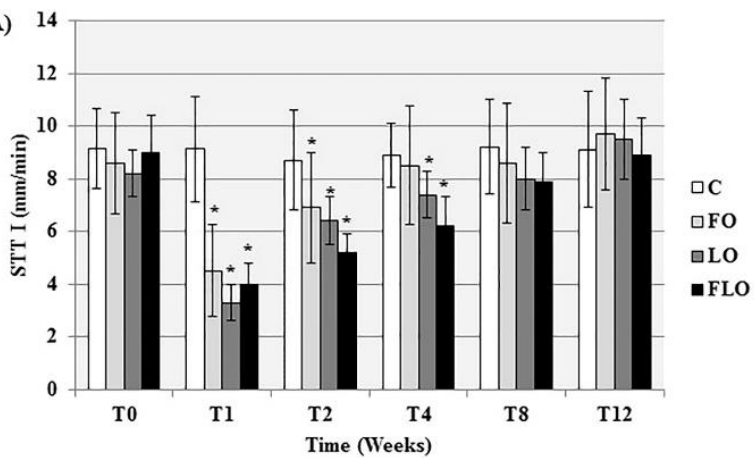

(B)

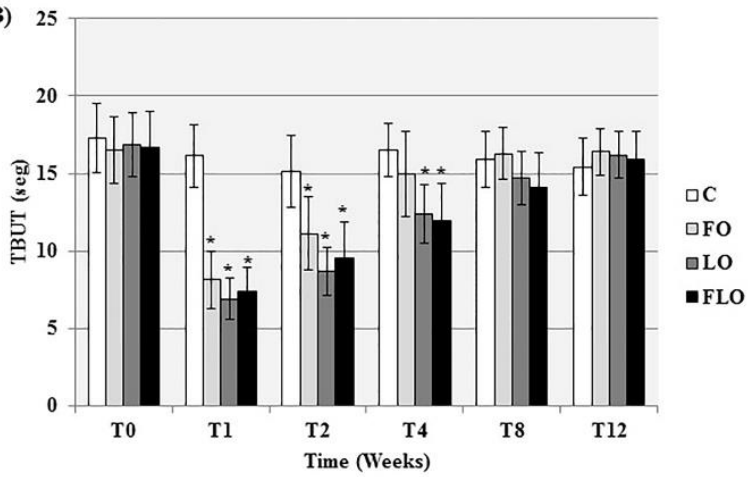

Fig. 2. Means and standard deviations of the values obtained from the (A) Schirmer Tear Test (STT I) [Values $\leq 5 \mathrm{~mm} / \mathrm{min}$ (positive for KCS)] in $\mathrm{mm} / \mathrm{min}$ and (B) the tear film break-up time (TBUT) test [TBUT $\leq 10$ seconds (positive for KCS)] in seconds in rabbits with experimentally induced keratoconjunctivitis sicca (KCS) that were subjected to oral treatment with fish oil (FO), linseed oil (LO) or a combination of fish oil and linseed oil (FLO), and the control group (C). *: $\mathrm{P}<0.05$ (Tukey's test for comparisons with the control group at every time point). 

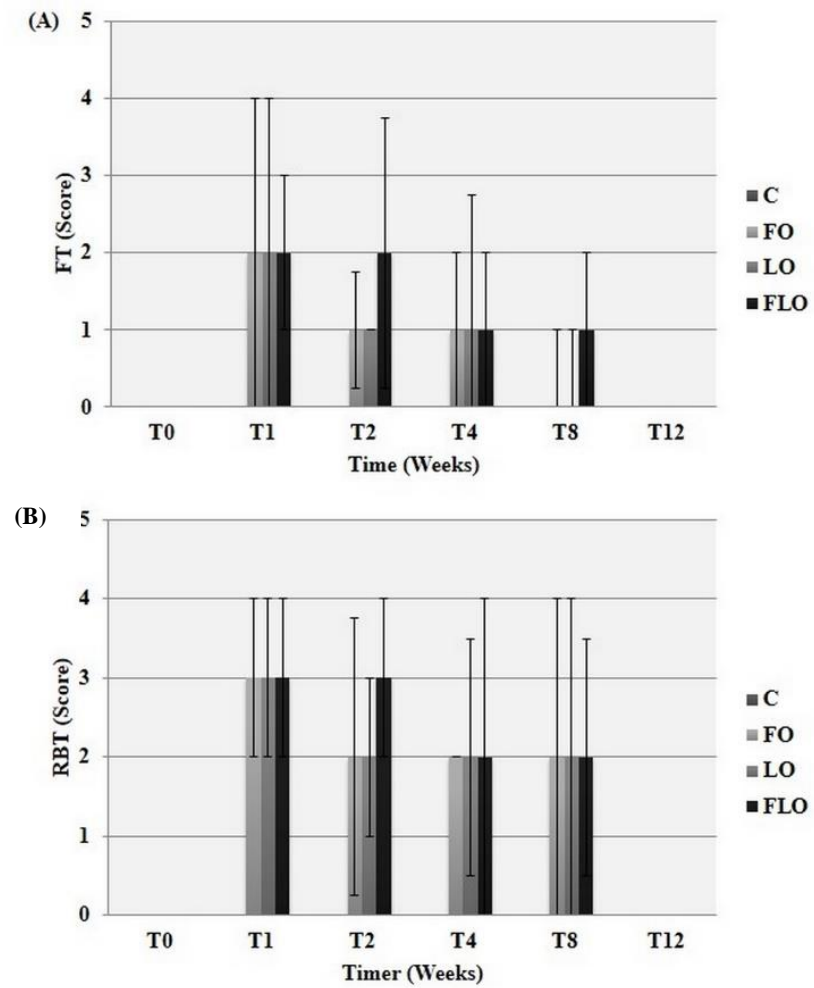

Fig. 3. Median and interquartile deviations of the values for the (A) fluorescein test (FT) [(0): without staining; (1): one stained quadrant; (2): two stained quadrants; (3): three stained quadrants; and (4): four stained quadrants] and (B) Rose Bengal Test (RBT) [(0): without staining; (1): only the conjunctiva was stained; (2): only the cornea was stained; and (3): both the conjunctiva and the cornea were stained] in rabbits with experimentally induced keratoconjunctivitis sicca (KCS) that were subjected to oral treatment with fish oil (FO), linseed oil (LO) or a combination of fish oil and linseed oil (FLO), and the control group (C).

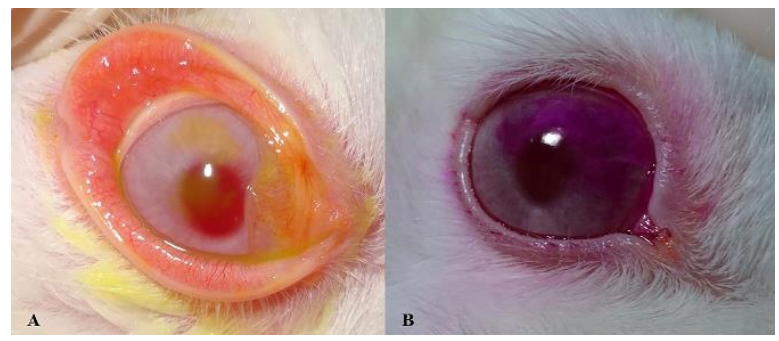

Fig. 4. (A) Right eye of rabbit No. 5 in the FLO group at T4 showing a conjunctival injection and a positive FT with superficial staining of the cornea. (B) Right eye of rabbit No. 2 in the LO group at T2 demonstrating a positive RBT result with staining in the superior quadrant of the cornea.

\section{Discussion}

In this study, orally administered FO and LO improved the clinical signs of experimentally induced KCS in rabbits, increased STT I and TBUT scores. This was possibly because these oils are rich in $\omega-3$ and $\omega-6$ fatty acids, which inhibit the formation of pro-inflammatory eicosanoids and increase the formation of antiinflammatory mediators, primarily including EPA, DHA, PGE1 and TXA1 (Oomah, 2001; Covington, 2004).

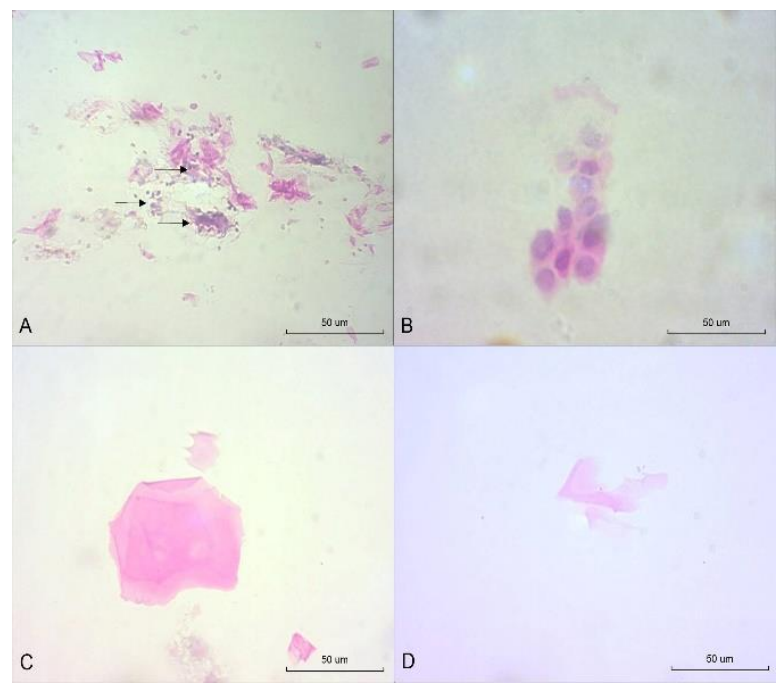

Fig. 5. Cytological smears: (A) T1 in the FLO group (OS): anucleate squamous cells and inflammatory cells in the conjunctiva (arrows) (hematoxylin-eosin (HE), 400x). (B) T1 in the FLO group (OS): metaplastic cells in the conjunctiva (HE, 400x). (C) $\mathrm{T} 1$ in the FLO group (OS): anucleate squamous cells of the cornea (HE, 400x). (D) T4 in the FO group (OD): neutral mucus (PAS-alcian blue, 400x). 


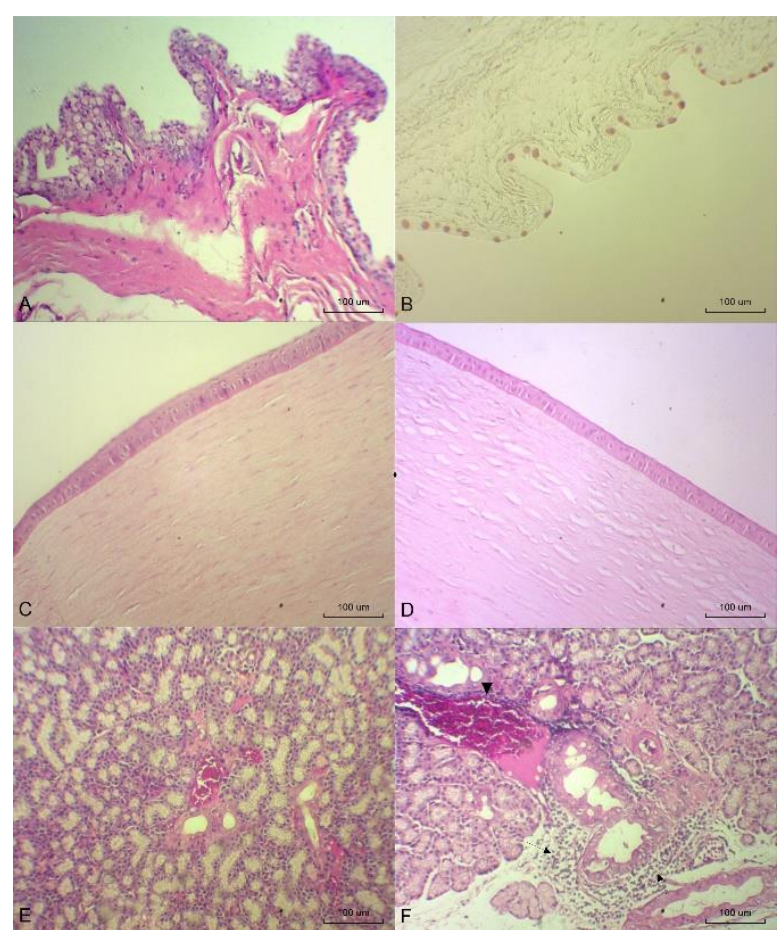

Fig. 6. Photomicrographs of the conjunctiva, cornea and lacrimal gland at T12. (A) LO group (OD): conjunctiva with large numbers of goblet cells (hematoxylin-eosin (HE), 100x). (B) LO group (OS): goblet cells (PAS-alcian blue, 100x). (C) LO group (OD): normal cornea (HE, 100x). (D) LO group (OD): cornea with edema (HE, 100x). (E) FO group (OD): normal lacrimal gland (HE, 100x). (F) FLO group (OS): lacrimal gland with inflammatory infiltration (arrows) and vascular congestion (arrowhead) (HE, 100x).

The consumption of LO and FO has also been demonstrated to control inflammation by decreasing the production of agents that promote inflammatory processes (e.g., cytokines, TNF and interleukin-1 $\beta$ ) in studies involving healthy volunteers and patients with rheumatoid arthritis who were provided with dietary supplements containing these EFAs (James et al., 2000).

A recent veterinary study concluded that LO, administered either orally or topically, was effective in treating induced KCS in rabbits (Neves et al., 2013), although the experimental study used in this KCS induction protocol, topical atropine, was combined with third eyelid gland removal, which was different from our study, in which KCS was induced using topical atropine alone. However, these data (Neves et al., 2013), show that even when a more aggressive induction protocol is used that results in the occurrence of severe ulcers, such as keratomalacia, which was not observed in our study, linseed oil was considered effective for treating KCS in rabbits.

Our study's result are also in agreement with the findings reported in a study that used a topical formulation containing $\omega-6$ and $\omega-3$ in mice that were experimentally induced to develop KCS. In that study, the mice demonstrated improvement in the inflammatory symptoms associated with KCS (Rashid et al., 2008). Another study by $\mathrm{He}$ and Bazan, also reported that the topical application of lipid derivatives containing $\omega-3$, such as DHA, prevented complications related to KCS, such as erosion and corneal ulcers (He and Bazan, 2010).

In the present study, the FO group demonstrated earlier improvement in the analyzed parameters, possibly because this oil already contains EPA and DHA as a result of the ingestion by fish of marine plants that contain synthesized $\omega-3$, whereas for LO, it is necessary to convert ALA to EPA and DHA (Jenkins and Josse, 2008). The human body can convert approximately $5 \%$ to $10 \%$ of ALA to EPA, but less than $2 \%$ to 5\% of ALA it is converted to DHA (Burdge, 2006; Rand and Asbell, 2011). In rabbits, dogs and cats, the conversion of ALA to EPA and DHA is also limited (Bauer, 2008; Ander et al., 2010).

In the present study, the combined oil treatment (the FLO group) did not result in additional benefits, demonstrating that this combination does not maximize the effects of these oils. Additionally, in this group, the number of goblet cells was lower. Because these cells are responsible for producing mucin in pre-tear film, which aids ocular lubrication, a reduction in the number of these cells could lead to deficient corneal lubrication (Davidson and Kuonen, 2004). In the cytological evaluation, the FLO group presented a higher incidence of inflammation and a higher number of keratinized corneal epithelial cells than were observed in the other groups at all time points. These data indicate that the cells produced more keratin to protect themselves from destruction. In the histopathological evaluation, edema and congestion were higher in the conjunctiva in the FLO group, possibly as a result of an inflammatory process (Kunert et al., 2002; Davidson and Kuonen, 2004; Robbins et al., 2015).

The combination therapy (the FLO group) was not as effective as either FO or LO alone, and a possible explanation for this result is that excess $\omega-3$ and $\omega-6$ results in an improper ratio of these two EFAs. This hypothesis was also suggested by Neves et al. (2013) that showed that LO, whether administered orally or topically, was effective in treating experimentally induced KCS in rabbits, while a combination of oral and topical LO did not result in additional benefits beyond those that were obtained from delivering $\mathrm{LO}$ via a single route.

We conclude that orally administered FO and LO effectively improved clinical signs of KCS in rabbits, possibly because these oils are rich in $\omega-3$ and $\omega-6$, which are both considered to be natural antiinflammatory agents. FO induced these benefits earlier, possibly because it contains preformed EPA and DHA. 
Combining the oils did not provide additional benefits. These results contribute to our ability to develop new oral formulations as an adjuvant therapy for $\mathrm{KCS}$ in the future.

\section{Acknowledgments}

We would like to thank the Post Graduate Program in Animal Science of UNOESTE, Laboratory Ophthalmos - SP and Laboratory Organnact - PR, for the donation of some of the materials necessary for the execution of the experiment.

\section{Conflict of interest}

The authors declare that they have no competing interests.

\section{References}

Ander, B.P., Edel, A.L., McCullough, R., RodriguezLeyva, D., Rampersad, P., Gilchrist, J.S.C., Lukas, A. and Pierce, G.N. 2010. Distribution of omega-3 fatty acids in tissues of rabbits fed a flaxseedsupplemented diet. Metabolism 59, 620-627.

Barabino, S., Rolando, M., Camicione, P., Ravera, G., Zanardi, S., Giuffrida, S. and Calabria, G. 2003. Systemic linoleic and g-linolenic acid therapy in dry eye syndrome with an inflammatory component. Cornea 22, 97-101.

Bauer, J.E. 2008. Essential fatty acid metabolism in dogs and cats. Rev. Bras. Zootecn. 37, 20-27.

Berdoulay, Y.A., English, R.V. and Naldelstein, B. 2005. Effect of topical $0.02 \%$ tacrolimus aqueous suspension on tear productin in dog with Keratoconjunctitis Sicca. Vet. Opthalmol. 8, 225232.

Burdge, G.C. 2006. Metabolism of alpha-linoleic acid in humans. Prostag. Leukotr. Ess. 75, 161-168.

Burgalassi, S., Panichi, L., Chetoni, P., Saettone, M.F. and Boldrini, E. 1999. Development of a simple dry eye model in the albino rabbit and evaluation of some tear substitutes. Ophthalmic Res. 31, 229-235.

Chong, E.W., Kreis, A.J., Wong, T.Y., Simpson, J.A. and Guymer, R.H. 2008. Dietary-3 fatty acid and fish intake in the primary prevention of age related macular degeneration. A systematic review and meta-analysis. Arch. Ophthalmol. 126, 826-833.

Covington, M.B. 2004. Omega-3 fatty acids. Am. Fam. Physician. 70, 133-140.

Davidson, H.J. and Kuonen, V.J. 2004. The tear film and ocular mucin (Review). Vet. Ophthalmol. 7, 7177.

El-Shazly, A.H., El-Gohrary, A.A., El-Shazly, L.H. and El-Hossary, G.G. 2008. Comparison between two cyclooxygenase inhibitors in an experimental dry eye model in albino rabbits. Acta. Pharm. 58, 163-173.

Grahn, B.H. and Storey, E.S. 2004. Lacrimomimetics and lacrimostimulants. Vet. Clin. North Am. Small Anim. Pract. 34, 739-753.
Harris, W.S. 2004. Fish oil supplementation: evidence for health benefits. Cleve. Clin. J. Med. 71, 208221.

He, J. and Bazan, H.E.P. 2010. Omega-3 fatty acids in dry eye and corneal nerve regeneration after refractive surgery. Prostag. Leukotr. Ess. 82, 319325.

Izci, C., Celik, I., Alkan, F., Ogurtan, Z., Ceylan, C., Sur, E. and Ozkan, Y. 2002. Histologic characteristics and local cellular immunity of the gland of the third eyelid after topical ophthalmic administration of $2 \%$ cyclosporine for treatment of dogs with keratoconjunctivitis sicca. Am. J. Vet. Res. 63, 688-694.

James, M.J., Gibson, R.A. and Cleland, L.G. 2000. Dietary polyunsaturated fatty acids and inflammatory mediator production. Am. J. Clin. Nutr. 71, 343-348.

Jenkins, D.J. and Josse, A.R. 2008. Fish oil and omega3 fatty acids. Can. Med. Am. J. 178, 150.

Kris-Etherton, P.M., Harris, W.S. and Appel, L.J. 2002. Fish consumption, fish oil, omega-3 fatty acids, and cardiovascular disease. Circulation 106, 2747-2757.

Kunert, K.S., Tisdale, A.S. and Gipson, I.K. 2002. Goblet cell numbers and epithelial proliferation in the conjunctiva of patients with dry eye syndrome treated with cyclosporine. Arch. Ophthalmol. 120, 330-337.

Maggs, D.J. 2008. Basic diagnostic techiniques. In Slater's Fundamentas of Veterinary Ophthalmology, Eds., Maggs, D.J., Miller, P.E. and Ofri, R. Saunders Elsevier, St. Louis, pp: 81-106.

Mahaffey, K. 2004. Fish and shellfish as dietary sources of methylmercury and the omega-3 fatty acids, eicosahexaenoic acid and docosahexaenoic acid: risks and benefits. Environ. Res. 95, 414-428.

McGinnigle, S., Naroo, S.A. and Eperjesi, F. 2012. Evaluation of dry eye. Surv. Ophthalmol. 7, 293316.

Miller, P.E. 2008a. Structure and function of the eye. In Slater's Fundamentas of Veterinary Ophthalmology, Eds., Maggs, D.J., Miller, P.E. and Ofri, R. Saunders Elsevier, St. Louis, pp: 1-19.

Miller, P.E. 2008b. Lacrimal system. In Slater's Fundamentas of Veterinary Ophthalmology, Eds., Maggs, D.J., Miller, P.E. and Ofri, R. Saunders Elsevier, St. Louis, pp: 157-174.

Mozaffarian, D. and Rimm, E.B. 2006. Fish intake, contaminants, and human health: evaluating the risks and the benefits. J. Am. Med. Assoc. 296, 1885-1899.

Neves, M.L., Yamasaki, L., Sanches, O.C., Amaral, M.S.P., Stevanin, H., Giuffrida, R., Candido, E.R., Goes, J.E., Zulim, L.F.C., Schweigert, A., Fukui, R.M., Meirelles, C.C, Sasaki, C.A. and Andrade, S.F. 2013. Use of linseed oil to treat experimentally 
induced keratoconjunctivitis sicca in rabbits. $\mathrm{J}$. Ophthalmol. Inflamm. Infect. 3, 4.

Oehlenschläger, J. 2012. Seafood: nutritional benefits and risk aspects. Int. J. Vitam. Nutr. Res. 82, 168176.

Ofri, R., Lambrou, G.N., Allgoewer, I., Graenitz, U., Pena, T.M., Spiess, B.M. and Latour, E. 2009. Clinical evaluation of pimecrolimus eye drops for treatment of canine keratoconjunctivitis sicca: a comparison with cyclosporine A. Vet. J. 179(1), 7077.

Oomah, B.D. 2001. Flaxseed as functional source. J. Sci. Food Agric. 81, 889-894.

Rand, A.L. and Asbell, P.A. 2011. Current Opinion in Ophthalmology Nutritional Supplements for Dry Eye Syndrome. Curr. Opin. Ophthalmol. 22, 279282.

Rashid, S., Jin, Y., Ecoiffer, T., Barabino, B., Schaumberg, D.A. and Dana, M.R. 2008. Topical omega-3 and omega- 6 fatty acids for treatment of dry eye. Arch. Ophthalmol. 126, 210-225.

Robbins, S.L., Cotran, R.S. and Kumar, V. 2015. Robbins \& Cotran: Pathologic Basis of Disease. Saunders Elsevier, St. Louis.

Roncone, M., Bartlett, H. and Eperjesi, F. 2010. Essential fatty acids for dry eye: A review. Cont. Lens Anterior Eye 33, 49-54.

Sgrignoli, M.R., Yamasaki, L., Sanches, O.C.,
Giuffrida, R., Ricci, C.L., Santos, G.C., Valle, H.F.D., Zulim, L.F.C., Silva, D.A., Basso, K.M., Silva, M.C.A. and Andrade, S.F. 2013. Comparison of topical $0.03 \%$ tacrolimus in almond and linseed oil to treat experimentally induced keratoconjunctivitis sicca in rabbits. Int. $\mathrm{J}$. Ophthalmic. Pathol. 2, 3.

Shafaa, M.W., El Shazly, L.H., El Shazly, A.H., El Gohary, A.A. and El Hossary, G.G. 2011. Efficacy of topically applied liposome-bound tetracycline in the treatment of dry eye model. Vet. Opthalmol. 14, 18-25.

Stevenson, W., Chauhan, S.K. and Dana, R. 2012. Dry eye disease: an immune-mediated ocular surface disorder. Arch. Ophthalmol. 130, 90-100.

Sullivan, B.D., Cermak, J.M., Sullivan, R.M., Papas, A.S., Evans, J.E., Dana, M.R. and Sullivan, D.A. 2002. Correlation between nutrient intake and the polar lipid profiles of meibomian gland secretions in women with Sjogren's syndrome. Adv. Exp. Med. Biol. 506, 441-447.

Wei, X.E., Markoulli, M., Zhao, Z. and Willcox, M.D. 2013. Tear film break-up time in rabbits. Clin. Exp. Optom. 96, 70-75.

Wojtowicz, J.C., Butovich, I. and Uchiyama, E. 2011. Pilot, prospective, randomized, double-masked, placebo-controlled clinical trial of an omega-3 supplement for dry eye. Cornea 30, 308-314. 